University of South Carolina

Scholar Commons

2-15-2004

\title{
Electronic Structure of Nuclear-Spin-Polarization-Induced Quantum Dots
}

Yuriy V. Pershin Dr

University of South Carolina - Columbia, pershin@physics.sc.edu

Follow this and additional works at: https://scholarcommons.sc.edu/phys_facpub

Part of the Physics Commons

Publication Info

Published in Physical Review B, ed. Gene D. Sprouse, Volume 69, Issue 8, 2004, pages

085314-1-085314-5.

Pershin, Y. V. (2004). Electronic structure of nuclear-spin-polarization-induced quantum dots. Physical Review B, 69(8), 085314-1 - 085314-5. DOI: 10.1103/PhysRevB.69.085314

(c) Physical Review B, 2004, American Physical Society

This Article is brought to you by the Physics and Astronomy, Department of at Scholar Commons. It has been accepted for inclusion in Faculty Publications by an authorized administrator of Scholar Commons. For more information, please contact digres@mailbox.sc.edu. 


\title{
Electronic structure of nuclear-spin-polarization-induced quantum dots
}

\author{
Yu. V. Pershin \\ Center for Quantum Device Technology, Department of Physics, Clarkson University, Potsdam, New York 13699-5820, USA \\ and Grenoble High Magnetic Fields Laboratory, Max-Planck-Institute für Festkörperforschung and CNRS,
}

Boite Postale 166, F-38042 Grenoble Cedex 9, France

(Received 30 April 2003; published 20 February 2004)

\begin{abstract}
We study a system in which electrons in a two-dimensional electron gas are confined by a nonhomogeneous nuclear-spin polarization. The system consists of a heterostructure that has nonzero nuclei spins. We show that in this system electrons can be confined into a dot region through a local nuclear-spin polarization. The nuclear-spin-polarization-induced quantum dot has interesting properties indicating that electron energy levels are time dependent because of the nuclear-spin relaxation and diffusion processes. Electron confining potential is a solution of diffusion equation with relaxation. Experimental investigations of the time dependence of electron energy levels will result in more information about nuclear-spin interactions in solids.
\end{abstract}

DOI: 10.1103/PhysRevB.69.085314

PACS number(s): 73.23. $-\mathrm{b}, 72.25 .-\mathrm{b}, 75.40 . \mathrm{Gb}$

\section{INTRODUCTION}

The theoretical and experimental researches of quantum dots have attracted much attention in recent years. ${ }^{1}$ Quantum dots are usually fabricated experimentally by applying lithographic and etching techniques to impose a lateral structure onto an otherwise two-dimensional electron system. Lateral structures introduce electrostatic potentials in the plane of the two-dimensional electron gas, which confines the electrons to a dot region. The energy levels of electrons in such quantum dots are fully quantized like in an atom. In such electrically confined quantum dots the confining potential can be well represented by a parabolic potential.

Another method of low-dimensional structure fabrication consists of the application of spatially inhomogeneous magnetic fields. Several alternative magnetic structures that were subsequently realized experimentally have been proposed. Among them magnetic dots using a scanning tunneling microscope lithographic technique, ${ }^{2}$ magnetic superlattices by the patterning of ferromagnetic materials integrated by semiconductors, ${ }^{3}$ type-II superconducting materials deposited on conventional heterostructures, ${ }^{4}$ and nonplanar twodimensional electron gas (2DEG) systems grown by a molecular-beam epitaxy. ${ }^{5}$ Such systems were studied theoretically in a series of papers by different authors. ${ }^{6-14}$

In the present paper we study a quantum dot system which is different from the quantum dot systems discussed above: (1) the electrons are confined through local nuclearspin polarization, (2) the confinement potential is inherently nonparabolic and time dependent, it is a solution of the diffusion equation when considering relaxation, and (3) the dot contains electrons with only one spin direction. Such system was proposed for the first time in Ref. 15. However, the properties of nuclear-spin-polarization-induced quantum dots (NSPIQD) have not been considered thus far and this is the motivation behind the present investigation. In our calculations we use some ideas from Ref. 16, where a nuclear-spinpolarization-induced quantum wire was proposed and investigated.

Electron and nuclear spins interact via the contact hyperfine interaction. Once the nuclear spins are polarized, the charge-carrier spins feel the effective hyperfine field $\mathbf{B}_{h f}$ which lifts the spin degeneracy. The maximum nuclear field in GaAs can be as high as $B_{h f}=5.3 \mathrm{~T}$ in the limit that all nuclear spins are fully polarized. ${ }^{17}$ This high level of nuclearspin polarization has been achieved experimentally. For example, the optical pumping of nuclear spins in 2DEG has demonstrated nuclear-spin polarization of the order of $90 \%{ }^{18}$ A similarly high polarization has been created by quantum Hall edge states (85\%). ${ }^{19}$ The spin splitting due to such a hyperfine magnetic field is comparable to the Fermi energy of 2DEG. It is important to note that the hyperfine field does not manifest itself magnetically due to the smallness of the nuclear magnetic moments. The electrons in the region where nuclear spins are polarized will preferably occupy the energetically more favorable states with the spins opposite to $\mathbf{B}_{h f}$. Furthermore, the nuclear polarization acts on the electrons as the effective confining potential. This effective confining potential can be used to create different nanostructures with polarized electrons in them. An example of such a nanostructure-NSPIQD-is considered here. Moreover, it might be well to point out that the hyperfine interactions play an important role in a decoherence process of an electron spin confined in an usual quantum dot. This decoherence mechanism was analyzed recently. ${ }^{20-23}$

The proposed system is depicted in Fig. 1. The nuclear spins are polarized locally along the $z$-axis in plane of the

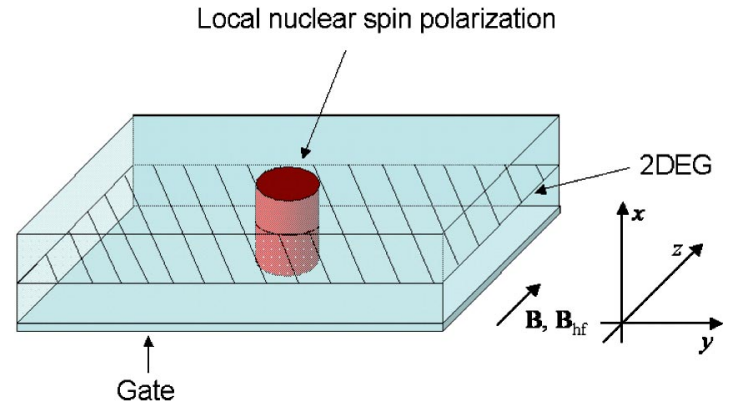

FIG. 1. The geometry of the proposed experiment: the NSPIQD is created in the region of intersection of the 2DEG with the local nuclear-spin polarization. 


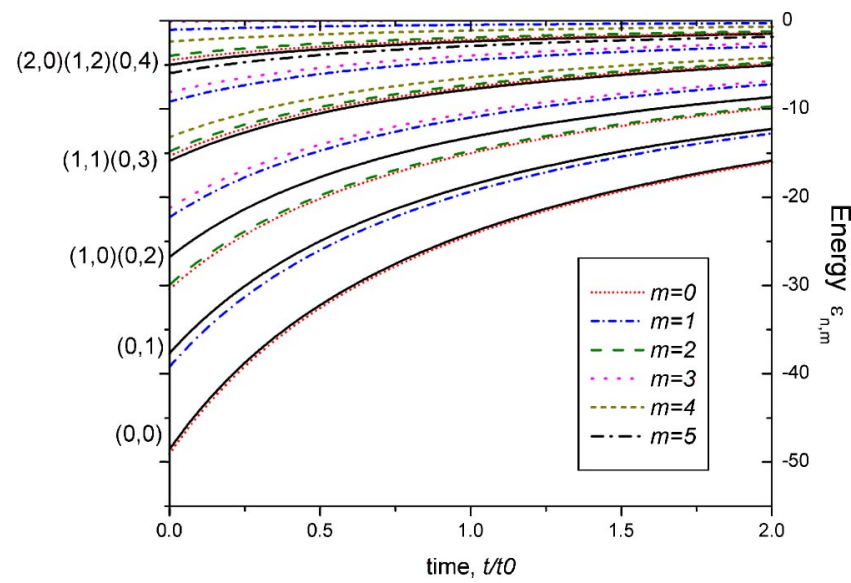

FIG. 2. Energy spectra of electrons in NSPIQD with initial halfwidth $d=1 \mu \mathrm{m}$ and $B_{h f}(r=0, t=0)=2.65 \mathrm{~T}$ as a function of time in the diffusion regime, $T_{1} / t_{0}=100$. The black solid lines are the energy levels for parabolic potential labeled by quantum numbers $(n, m)$ at the left. The other lines correspond to the energy levels for the Gaussian potential, the lines having a same color have the same quantum number $m$; the quantum number $n$ is equal to 0 for the lowest line of each color and increases by 1 for lines of the same color from bottom to top.

2DEG in heterostructure by any suitable experimental method. For example, the optical nuclear-spin polarization ${ }^{24-27}$ or the transport polarization ${ }^{28-30}$ can be used. The region where the nuclear spins are polarized is indicated by the cylinder in Fig. 1. It is assumed that the nuclear-spin polarization is homogeneous in $x$-direction perpendicular to the 2DEG. The NSPIQD is created in the region of intersection of the 2DEG with the region of local nuclear-spin polarization. The gate electrode below the 2DEG is used to control the number of electrons in the NSPIQD. Moreover, the system is subjected to an external magnetic field along the $z$ axis.

The magnetic field is of importance to the nuclear-spin polarization process. A detailed experimental study of the optical nuclear-spin polarization was reported in Ref. 27. In this paper the level of nuclear-spin polarization was monitored versus magnetic field, temperature, and pump intensity in GaAs quantum well. It was found that the nuclear-spin polarization disappears at $B=0$ and increases rapidly with $B$ for $B<0.3 \mathrm{~T}$. The nuclear-spin polarization has a peak at $5 \mathrm{~K}$ and $B=2 \mathrm{~T}$, and decreases slowly with subsequent increase of $B$ (see Fig. 2 of Ref. 27). Disappearance of nuclear-spin polarization at $B=0$ can be related to the nuclear-spin relaxation due to the off-diagonal terms in dipole-dipole interaction Hamiltonian (p. 66 of Ref. 31). In a finite magnetic field nuclear Zeeman gap opens. As a consequence, the transitions generated by these terms become damped and, correspondingly, the nuclear-spin relaxation time increases. Thus, for an efficient optical nuclear-spin polarization the external magnetic field should be equal or higher than $0.3 \mathrm{~T}$. In such magnetic fields the nuclear-spin polarization is in one direction with applied magnetic field. ${ }^{27}$

Our paper is organized as follows. In Sec. II we discuss the properties of nonhomogeneous nuclear-spin polarization and calculate the evolution of initially created hyperfine-field profile which is taken, for simplicity, in the Gaussian form. Time dependence of the electron states in NSPIQD is studied in Sec. III. The conclusions of this investigation are presented in Sec. IV.

\section{HYPERFINE-FIELD PROFILE}

Let us assume that the method of optical nuclear-spin polarization is used ${ }^{24-27}$ to create a NSPIQD. To pattern a nanostructure it is proposed to illuminate the sample locally by, for example, putting a mask on it. The usual optical technique allows one to create the light beams of the width of the order of the wavelength $(\sim 500 \mathrm{~nm})$. By using near field optics the beamwidth can be sufficiently reduced $(\sim 100 \mathrm{~nm})$. Hence a $1-\mu \mathrm{m}$-size NSPIQD can be easily created by the modern experimental technique.

There are two main mechanisms leading to the time dependence of the hyperfine field: the nuclear-spin relaxation and the nuclear-spin diffusion. We assume that the initial nuclear-spin polarization is along the $z$ direction and homogeneous in the $x$ direction. Then the hyperfine-field evolution is described by the two-dimensional diffusion equation

$$
\frac{\partial B_{h f}}{\partial t}=D \Delta B_{h f}-\frac{1}{T_{1}} B_{h f},
$$

accounting for the relaxation processes. Here $D$ is the spindiffusion coefficient, $\Delta=\partial^{2} / \partial y^{2}+\partial^{2} / \partial z^{2}$ is a twodimensional Laplace operator, and $T_{1}$ is the nuclear-spin relaxation time..$^{31,32}$ The formal solution of Eq. (1) can be written as

$$
B_{h f}=e^{-t / T_{1}} \int G\left(\mathbf{r}-\mathbf{r}^{\prime}, t\right) B_{h f}\left(\mathbf{r}^{\prime}, t=0\right) d \mathbf{r}^{\prime} .
$$

Here $G\left(\mathbf{r}-\mathbf{r}^{\prime}, t\right)=e^{-\left(\mathbf{r}-\mathbf{r}^{\prime}\right)^{2} / 4 D t} / 4 \pi D t$ is the Green function of the diffusion equation and $B_{h f}\left(\mathbf{r}^{\prime}, t=0\right)$ is the initial hyperfine-field profile.

In this paper we consider NSPIQD having the cylindrical symmetry; that is, the hyperfine field $B_{h f}$ is a function of $r$, that is in $(y, z)$ plane. In the simplest case, we can assume the initial condition to be of the Gaussian form: $B_{h f}(r, 0)$ $=B_{0} \exp \left(-r^{2} / 2 d^{2}\right)$. The two parameters, $d$ and $B_{0}$, define the half-width and the amplitude of the initial distribution of the hyperfine field, respectively. Then the solution of Eq. (1) is

$$
B_{h f}(r, t)=B_{0} e^{-t / T_{1}}\left(1+\frac{t}{t_{0}}\right)^{-1} e^{-\left[r^{2} / 2 d^{2}\left(1+t / t_{0}\right)\right]},
$$

where $t_{0}=d^{2} / 2 D$. The value of $t_{0}$ is the time it takes for $B_{h f}(0, t)$ to reduce by a factor of 2 from $t=0$ due to the nuclear-spin diffusion. The nuclear-spin relaxation time $T_{1}$ in semiconductors at sufficiently low temperatures is rather long. It varies from several hours to a few minutes. ${ }^{25}$ The available experimental values for the diffusion coefficient are $D \sim 10^{-13} \mathrm{~cm}^{2} \mathrm{~s}^{-1}$ for ${ }^{75} \mathrm{As}$ in bulk $\mathrm{GaAs}^{33}$ and $D$ 
$=10^{-14} \mathrm{~cm}^{2} \mathrm{~s}^{-1}$ in $\mathrm{Al}_{0.35} \mathrm{Ga}_{0.65} \mathrm{Ass}^{34}$ For $d=1$ and $5 \mu \mathrm{m}$ taking $D=10^{-13} \mathrm{~cm}^{2} \mathrm{~s}^{-1}$ we have $t_{0}=5 \times 10^{4}, 1.25$ $\times 10^{6} \mathrm{~s}$.

\section{ENERGY SPECTRUM}

The microscopic description is based on the following Hamiltonian:

$$
H=-\frac{\hbar^{2}}{2 m^{*}} \Delta+\frac{1}{2} g^{*} \mu_{B} \sigma\left[\mathbf{B}_{h f}(r, t)+\mathbf{B}\right]+U(x),
$$

where $m^{*}$ is the electron effective mass, $g^{*}$ is the effective electron $g$ factor $\left(g_{\mathrm{GaAs}}^{*}=-0.44\right), \mu_{B}$ is the Bohr magneton, $\sigma$ is the vector of Pauli matrices, $\mathbf{B}_{h f}$ is given by Eq. (3), $\mathbf{B}$ is the magnetic field in $z$ direction, and $U(x)$ is the 2DEG confining potential. We suppose, as is usually done for the 2DEG, that only the lowest subband, corresponding to the confinement in $x$ direction, is occupied and we can ignore the higher subbands. Thus, we omit $x$ dependence of the wave function in the following. The time scale introduced by a nuclear-spin system is several orders of magnitude larger than the time scale of typical electron equilibration processes. In such a case the conduction electrons see a quasiconstant nuclear field. This simplifies calculation by avoiding the complications which would appear when solving the Schrödinger equation with the time dependence due to polarized nuclei. We take into account the electrons of only one spin direction (for which the effective potential is attractive). Since $\mathbf{B}_{h f}$ and $\mathbf{B}$ are unidirectional, the effect of $\mathbf{B}$ is just a uniform energy shift for electrons with one spin direction. Therefore, the term with $\mathbf{B}$ in Hamiltonian (4) will be omitted in what follows.

We note that Hamiltonian (4) implies that only $z$ part of the contact hyperfine interaction between nuclear spin and electron spin is taken into account. This is a good approximation in finite magnetic fields considered here because of restrictions imposed by the energy conservation, i.e., by the large difference between the electron and nuclear Zeeman splitting. At finite temperatures, the energy conservation law can be satisfied by absorbing a phonon in the flip-flop process of nuclear and electron spins. ${ }^{35}$ However, this mechanism is suppressed at low temperatures and consideration of this process is out of the scope of this paper.

The one-electron eigenvalue problem with the attractive Gaussian potential [Eq. (3)] does not admit analytical solutions. Different approximate methods ${ }^{36-40}$ were implemented to solve this problem. In the present paper, an analytical solution of the Schrödinger equation is found within the parabolic approximation of the hyperfine field: ${ }^{36}$

$$
\widetilde{B}_{h f}=a-b r^{2}
$$

connected with Eq. (3) by the relations

$$
\widetilde{B}_{h f}(0, t)=B_{h f}(0, t)
$$

and

$$
\left.\widetilde{B}_{h f}^{\prime \prime}(r, t)\right|_{r=0}=\left.B_{h f}^{\prime \prime}(r, t)\right|_{r=0}
$$

Here $r_{0}^{2}=a / b$. Eq. (6) connects the depth of potentials, Eq. (7) provides equal second derivatives for the two fields at $r$ $=0$. From Eqs. (6) and (7) we obtain $a=B_{0}\left[e^{-t / T_{1} / 1}\right.$ $\left.+\left(t / t_{0}\right)\right]$ and $b=B_{0}\left[e^{-t / T_{1} / 2} d^{2}\left(1+t / t_{0}\right)^{2}\right]$. The energy spectrum for the parabolic potential (5) in units of $E_{0}$ $=\hbar^{2} / 2 m * d^{2}$ is given by

$$
\begin{aligned}
\varepsilon_{n, m}= & -\frac{g^{*} \mu_{B} B_{0}}{2 E_{0}} \frac{e^{-t / T_{1}}}{1+\frac{t}{t_{0}}}+\sqrt{\frac{g^{*} \mu_{B} B_{0}}{E_{0}}} \frac{e^{-t / 2 T_{1}}}{1+\frac{t}{t_{0}}} \\
& \times(2 n+|m|+1),
\end{aligned}
$$

where $n=0,1, \ldots$ and $m=0, \pm 1, \ldots$.

The exact solution of the Schrödinger equation with the Gaussian profile of the hyperfine field [Eq. (3)] was found numerically. Due to the cylindrical symmetry of the problem, the wave function can be written as $\psi(\rho, \phi)$ $=(1 / \sqrt{2 \pi}) e^{i m \phi} R(\rho)$. The equation for the radial part $R(r)$ of wave function has a form

$$
\left[\frac{1}{x} \frac{d}{d x} x \frac{d}{d x}-\frac{m^{2}}{x^{2}}+\gamma \frac{B_{h f}(x, t)}{B_{h f}(0,0)}+\varepsilon_{n, m}\right] R_{n, m}=0,
$$

where $x=r / d$ is the dimensionless coordinate and $\gamma$ $=g^{*} \mu_{B} B_{h f}(0,0) / 2 E_{0}$. For $d=1$ and $5 \mu \mathrm{m}$, taking $m^{*}$ $=0.067 m_{e}$, we have $E_{0}=0.57 \times 10^{-3}, 0.023 \times 10^{-3} \mathrm{meV}$; for $B_{h f}(0,0)=2.65$ (50\% nuclear-spin polarization) and $5.3 \mathrm{~T}$ (100\% nuclear-spin polarization) corresponding energies are $\frac{1}{2} g^{*} \mu_{B} B_{h f}(0,0)=3.4 \times 10^{-2}$ and $6.8 \times 10^{-2} \mathrm{meV}$. We have used the shooting method to solve Eq. (9), subjecting the solution to the following boundary conditions: $R_{n, m}(\rho \rightarrow 0)$ $=\rho^{|m|}$ and $R_{n, m}(\rho \rightarrow \infty)=0$. The results of the numerical calculations are presented below.

The time dependence of the electron energy levels in the NSPIQD is determined by the time dependence of the confining hyperfine field. There are two characteristic times in the problem: the diffusion characteristic time $t_{0}$ and the relaxation characteristic time $T_{1}$. We can distinguish the diffusive regime, when $t \sim t_{0} \ll T_{1}$, the intermediate regime, $t$ $\sim t_{0} \sim T_{1}$, and the relaxation regime, $t \sim T_{1} \ll t_{0}$. Here $t$ is the observation time.

Figure 2 shows the time dependence of the electron energy levels for the Gaussian and parabolic potentials in the diffusion regime. We emphasize that the parabolic potential can be regarded as a good approximation of the Gaussian potential only for the ground state. The excited-state energy levels for the parabolic potential reveal large deviations from those for the Gaussian potential, which manifest in the degeneracy of states and in the shift of levels. This result is qualitatively similar to those obtained for 3D Gaussian and parabolic potential. ${ }^{36}$ However, time dependence of energy levels for both potentials show quite similar behavior. The number of energy levels in NSPIQD remains constant, whereas their depth decreases. From Eq. (8) it follows that in the diffusion regime the time dependence of the energy levels in the parabolic potential is $\varepsilon_{n, m}(t)=\left[\varepsilon_{n, m}(0) / 1\right.$ $\left.+\left(t / t_{0}\right)\right]$. It can be shown that the energy levels in the 


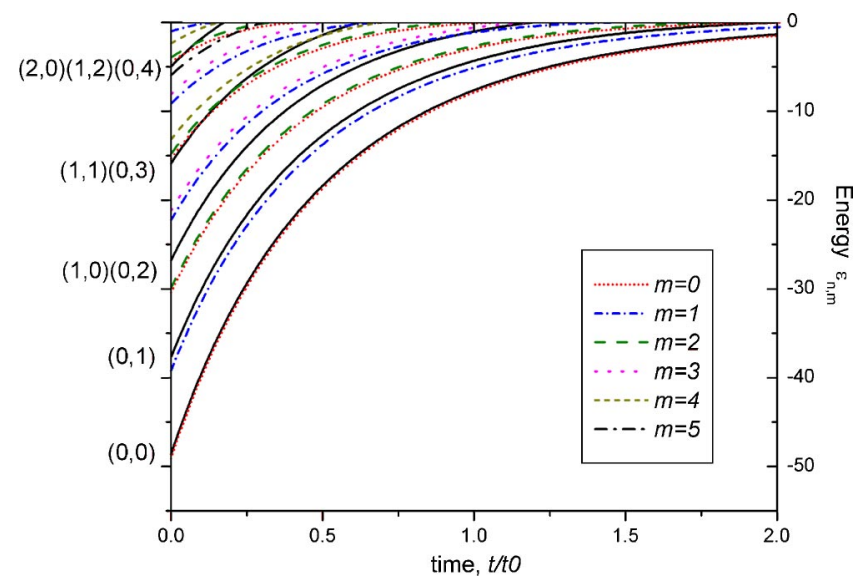

FIG. 3. Energy spectra of electrons in NSPIQD as a function of time in the intermediate regime, $T_{1} / t_{0}=1$. The parameters of calculations and labeling of levels are as in Fig. 2.

Gaussian potential have the same time dependence. Substituting Eq. (3) into Eq. (9) and introducing the variable $\xi$ as $x=\xi \sqrt{1+t / t_{0}}$ we obtain

$$
\left[\frac{1}{\xi} \frac{d}{d \xi} \xi \frac{d}{d \xi}-\frac{m^{2}}{\xi^{2}}+\gamma e^{-\xi^{2} / 2}+\left(1+\frac{t}{t_{0}}\right) \varepsilon_{n, m}\right] R_{n, m}=0 .
$$

The time-dependent factor, $\left(1+t / t_{0}\right)$, appears in Eq. (10) only as a product with $\varepsilon_{n, m}$ thus proving the statement.

Figures 3 and 4 show the results obtained for the intermediate and relaxation regimes. On the contrary, the number of the energy levels in NSPIQD decreases in time in these regimes. This decrease occurs on the scale of $T_{1}$. We cannot explicitly obtain time dependence of energy levels for the Gaussian potential in these regimes. The parabolic approximation of the hyperfine field serves as a good approximation again only for the ground energy level. The evolution of excited-state energy levels in the Gaussian and in the parabolic potentials are different: the lifetimes of energy levels

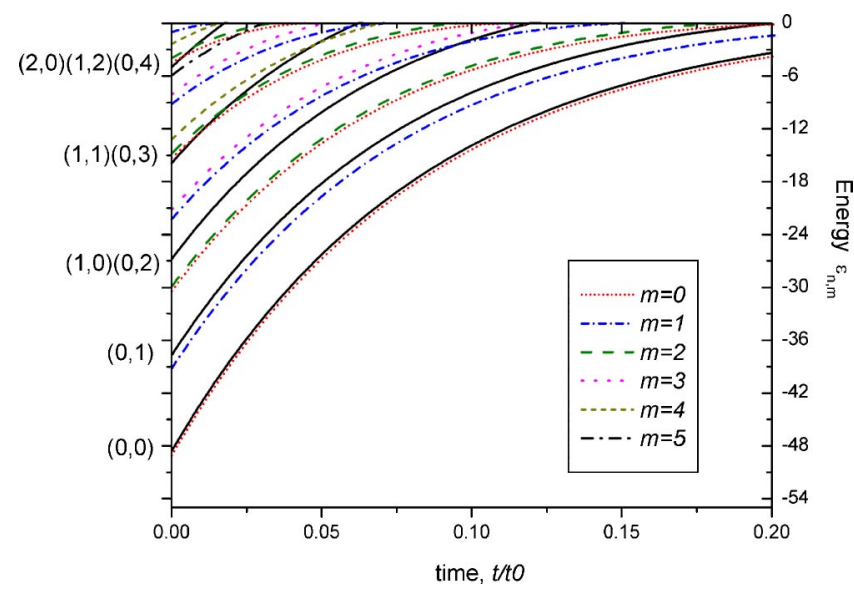

FIG. 4. Energy spectra of electrons in NSPIQD as a function of time in the relaxation regime, $T_{1} / t_{0}=0.1$. The parameters of calculation and labeling of levels are as in Fig. 2. obtained in the case of the parabolic potential are shorter than in the case of the Gaussian potential.

It is important to know the lifetime of the NSPIQD. We can consider electron states in the NSPIQD up to the moment when the confining potential depth is more than the temperature. Consequently, the lifetime $t_{l}$ of the NSPIQD can be defined by the following condition: $\left|g^{*} \mu_{B} B_{h f}\left(0, t_{l}\right)\right| / 2=k_{B} T$, where $k_{B}$ is the Boltzmann constant and $T$ is the temperature. Using Eq. (3), we calculate time $t_{l}$ for two limiting cases: $T_{1} \ll t_{0}$ and $T_{1} \gg t_{0}$. In the first case (the relaxation regime), $t_{l} \sim T_{1} \ln \left|g^{*} \mu_{B} B_{0}\right| / 2 k_{B} T$. In the second case (the diffusion regime), $t_{l}$ $\sim t_{0}\left|g * \mu_{B} B_{0} / 2 k_{B} T-1\right|$. Time dependence of the half-width of NSPIQD is $d(t)=d \sqrt{1+t / t_{0}}$. Let us estimate it at $t=t_{l}$. For $T=30 \mathrm{mK}$ and $B_{0}=2.65 \mathrm{~T}$ we have $d\left(t_{l}\right)=d$ in the relaxation regime and $d\left(t_{l}\right)=3.6 d$ in the diffusion regime.

\section{CONCLUSIONS}

We have studied the electron energy levels of a NSPIQD created in the region of the intersection of a local nuclearspin polarization with a 2DEG. The properties of the NSPIQD are time dependent because of the nuclear-spin diffusion and relaxation. There are two characteristic time and three corresponding regimes: the diffusion regime, the intermediate regime, and the relaxation regime. In the diffusion regime, the number of electron energy levels remains constant with time. In the relaxation and intermediate regime, the number of electron energy levels decreases with time. Time dependence of the electron energy levels in the diffusion regime has a simple form. Since the characteristic diffusion time is proportional to the square of the NSPIQD radius at $t=0$, it is possible to create NSPIQDs operating in different regimes using the same experimental setup.

The numerical estimations allow us to conclude that the system under study can be realized experimentally. For a hyperfine field of just a few teslas, the experiment could be made at a temperature of the order of $10 \mathrm{mK}$. The modern experimental technique allows one to create a region with local nuclear-spin polarization of characteristic sizes $\gtrsim 100 \mathrm{~nm}$, making the NSPIQD having a small size. The spectroscopy of the NSPIQD could be used to obtain some information about nuclear-spin interactions in solids, for example, the nuclear-spin relaxation time and the nuclear-spin diffusion coefficient.

It should be pointed out that a simplified model was used in this paper to describe the single-electron states in the NSPIQD. We considered the influence of a nuclear-spinrelated hyperfine field on the electron states, whereas the electrons could also alter the nuclear-spin dynamics. The well-known examples of such phenomena are the indirect long-range nuclear-spin interaction, electron-assisted mechanisms of nuclear-spin relaxation and nuclear-spin precession in an effective field created by the electrons. ${ }^{31}$

Another important effect that was not mentioned so far is the Coulomb blockade. The Coulomb blockade effect is directly observed in transport measurements, when an addi- 
tional electron is added to a quantum dot. In the frame of constant-interaction model, ${ }^{1,41,42}$ the additional energy is $E$ $=e^{2} / C+\Delta E$, where $\Delta E$ is the energy difference between single-particle states, $e^{2} / C$ is the charging energy of a singleelectron charge $e$, on a capacitor $C$. For usual quantum dots the charging energy is of the order of $0.1 \mathrm{meV}^{41}$ for NSPIQD we believe that the charging energy is less because of the larger size of NSPIQD. In our model, the gate below 2DEG could be used to compensate the charging energy. Moreover, we propose to study NSPIQD by spectroscopy methods, which assume a fixed number of electrons in NSPIQD and involve transitions between single-particle states. In-depth study of these effects is beyond the scope of this paper. Results of such investigations will be published elsewhere.

\section{ACKNOWLEDGMENTS}

We acknowledge useful discussions with V. Privman, S. Shevchenko, and I. Vagner. This research was supported by the National Security Agency and the Advanced Research and Development Activity under Army Research Office Contracts Nos. DAAD-19-99-1-0342 and DAAD 19-02-1-0035, and by the National Science Foundation, Grants Nos. DMR0121146 and ECS-0102500.
${ }^{1}$ Quantum Dots, edited by L. Jacak, P. Hawrylak, and A. Wojs (Springer-Verlag, Berlin, 1998).

${ }^{2}$ M.A. McCord and D.D. Awschalom, Appl. Phys. Lett. 57, 2153 (1990).

${ }^{3}$ M.L. Leadbeater, S.J. Allen, Jr., F. DeRosa, J.P. Harbison, T. Sands, R. Ramesh, L.T. Florez, and V.G. Keramidas, J. Appl. Phys. 69, 4689 (1991); K.M. Krishnan, Appl. Phys. Lett. 61, 2365 (1992).

${ }^{4}$ S.J. Bending, K. von Klitzing, and K. Ploog, Phys. Rev. Lett. 65, 1060 (1990).

${ }^{5}$ M.L. Leadbeater, C.L. Foden, T.M. Burke, J.H. Burroughes, M.P. Grimshaw, D.A. Ritchie, L.L. Wang, and M. Pepper, J. Phys.: Condens. Matter 7, L307 (1995).

${ }^{6}$ F.M. Peeters and A. Matulis, Phys. Rev. B 48, 15166 (1993).

${ }^{7}$ I.S. Ibrahim, V.A. Schweigert, and F.M. Peeters, Phys. Rev. B 56, 7508 (1997).

${ }^{8}$ J. Reijniers, F.M. Peeters, and A. Matulis, Phys. Rev. B 59, 2817 (1999).

${ }^{9}$ For a review see F. M. Peeters and J. De Boeck, in Handbook of Nanostructured Materials and Nanotechnology, edited by H.S. Nalwa (Academic Press, New York, 1999), Vol. 3, p. 345.

${ }^{10}$ L. Solimany and B. Kramer, Solid State Commun. 96, 471 (1995).

${ }^{11}$ H.-S. Sim, K.-H. Ahn, K.J. Chang, G. Ihm, N. Kim, and S.J. Lee, Phys. Rev. Lett. 80, 1501 (1998).

${ }^{12}$ N. Kim, G. Ihm, H.-S. Sim, and K.J. Chang, Phys. Rev. B 60, 8767 (1999).

${ }^{13}$ N. Kim, G. Ihm, H.-S. Sim, and T.W. Kang, Phys. Rev. B 63, 235317 (2001).

${ }^{14}$ H.-S. Sim, G. Ihm, N. Kim, and K.J. Chang, Phys. Rev. Lett. 87, 146601 (2001).

${ }^{15}$ V. Fleurov, V.A. Ivanov, F.M. Peeters, and I.D. Vagner, Physica E (Amsterdam) 14, 361 (2002).

${ }^{16}$ Yu.V. Pershin, S.N. Shevchenko, I.D. Vagner, and P. Wyder, Phys. Rev. B 66, 035303 (2002).

${ }^{17}$ D. Paget, G. Lampel, B. Sapoval, and V.I. Safarov, Phys. Rev. B 15, 5780 (1977).

${ }^{18}$ G. Salis, D.D. Awschalom, Y. Ohno, and H. Ohno, Phys. Rev. B 64, 195304 (2001).

${ }^{19}$ D.C. Dixon, K.R. Wald, P.L. McEuen, and M.R. Melloch, Phys. Rev. B 56, 4743 (1997).
${ }^{20}$ I.A. Merkulov, Al.L. Efros, and M. Rosen, Phys. Rev. B 65, 205309 (2002).

${ }^{21}$ A. Khaetskii, D. Loss, and L. Glazman, Phys. Rev. Lett. 88, 186802 (2002); Phys. Rev. B 67, 195329 (2003).

${ }^{22}$ R. de Sousa and S. Das Sarma, Phys. Rev. B 67, 033301 (2003).

${ }^{23}$ A. Imamoglu, E. Knill, L. Tian, and P. Zoller, Phys. Rev. Lett. 91, 017402 (2003).

${ }^{24}$ G. Lampel, Phys. Rev. Lett. 20, 491 (1968).

${ }^{25}$ Optical Orientation, edited by F. Meier and B.P. Zakharchenya (Elsevier, Amsterdam, 1984).

${ }^{26}$ S.E. Barrett, R. Tycko, L.N. Pfeiffer, and K.W. West, Phys. Rev. Lett. 72, 1368 (1994); for a review see R. Tycko et al., Science 268, 1460 (1995).

${ }^{27}$ G. Salis, D.T. Fuchs, J.M. Kikkawa, and D.D. Awschalom, Phys. Rev. Lett. 86, 2677 (2001).

${ }^{28}$ B.E. Kane, L.N. Pfeiffer, and K.W. West, Phys. Rev. B 46, 7264 (1992).

${ }^{29}$ K.R. Wald, L.P. Kouwenhoven, P.L. McEuen, N.C. van der Vaart, and C.T. Foxon, Phys. Rev. Lett. 73, 1011 (1994).

${ }^{30}$ J. Strand, B.D. Schultz, A.F. Isakovic, C.J. Palmstrom, and P.A. Crowell, Phys. Rev. Lett. 91, 036602 (2003).

${ }^{31}$ C.P. Slichter, Principles of Magnetic Resonance, 3rd ed. (Springer-Verlag, Berlin, 1990).

${ }^{32}$ D. Wolf, Spin-Temperature and Nuclear-Spin Relaxation in Matter (Clarendon Press, Oxford, 1979).

${ }^{33}$ D. Paget, Phys. Rev. B 25, 4444 (1982).

${ }^{34}$ A. Malinovsky, M.A. Brand, and R.T. Harley, Physica E (Amsterdam) 10, 13 (2001).

${ }^{35}$ J.H. Kim, I.D. Vagner, and L. Xing, Phys. Rev. B 49, 16777 (1994).

${ }^{36}$ J. Adamowski, M. Sobkowicz, B. Szafran, and S. Bednarek, Phys. Rev. B 62, 4234 (2000).

${ }^{37}$ N. Bessis, G. Bessis, and B. Joulakian, J. Phys. A 15, 3679 (1982).

${ }^{38}$ C.S. Lai, J. Phys. A 16, L181 (1983).

${ }^{39}$ R.E. Crandall, J. Phys. A 16, L395 (1983).

${ }^{40}$ M. Cohen, J. Phys. A 17, L101 (1984).

${ }^{41}$ Mesoscopic Physics and Electronics, edited by T. Ando, Y. Arakawa, K. Furuya, S. Komiyama, and N. Nakashima (Springer, Berlin, 1998).

${ }^{42}$ L.P. Kouwenhoven, D.G. Austing, and S. Tarucha, Rep. Prog. Phys. 64, 701 (2001). 